\title{
Modelo de Transferencia Tecnológica desde la Ingeniería Biomédica: un estudio de caso
}

\author{
María T. De Ossa, John E. Londoño* y Alejandro Valencia-Arias \\ Facultad de Ciencias Económicas y Administrativas, Instituto Tecnológico Metropolitano, Calle 73 No. 76A - \\ 354 Vía al Volador, Medellín - Antioquia, Colombia. (e-mail: mariade7853@correo.itm.edu.co; \\ johnlondono@itm.edu.co; jhoanyvalencia@itm.edu.co) \\ ${ }^{*}$ Autor a quien debe ser dirigida la correspondencia.
}

Recibido Jun. 13, 2017; Aceptado Ago. 18, 2017; Versión final Oct. 6, 2017, Publicado Feb. 2018

\begin{abstract}
Resumen
Este estudio tuvo como objetivo adaptar un modelo de transferencia tecnológica asociado a los sistemas de rehabilitación en ingeniería biomédica en la ciudad de Medellín para el año 2016, mediante un análisis de caso. Se tomaron diez fundaciones que trabajaran con personas en situación de discapacidad, elegidas según su impacto social; dos organizaciones generadoras de ayudas técnicas de rehabilitación y una universidad con experiencia investigativa en ingeniería biomédica. De esta se tomó un prototipo, al cual se adaptó un modelo dinámico de transferencia. Una vez aplicado y validado, se evidenció la importancia de contextualizar las soluciones diseñadas para las personas discapacitadas, evaluando no sólo sus necesidades, sino también el contexto particular de cada individuo. Se concluye que los procesos ingenieriles de las universidades, deberán contar desde la generación de una idea innovadora con la presencia de las empresas y el Estado, para garantizar procesos de transferencia exitosos.
\end{abstract}

Palabras clave: transferencia tecnológica; ingeniería biomédica; sistemas de rehabilitación; universidadempresa

\section{Model of Technology Transfer from Biomedical Engineering: a case study}

\begin{abstract}
The objective of this study was to adapt a technology transfer model associated with rehabilitation systems in biomedical engineering in the city of Medellin in the year 2016, through a case study. Ten foundations were studied and chosen according to their social impact and their work with people with disabilities. In addition, two organizations that generate technical rehabilitation aids and a university with research experience in biomedical engineering were taken into account. From the latter, a prototype was taken, to which a dynamic transfer model was adapted. Once implemented and validated, the importance of contextualizing the solutions designed for the disabled people was evidenced, evaluating not only their needs, but also the particular context of each individual. It is concluded that the engineering processes of universities must include, from the generation of an innovative idea, the presence of companies and of institutions of the public sector to ensure successful transfer processes.
\end{abstract}




\section{INTRODUCCIÓN}

La ingeniería biomédica es reconocida como una profesión integradora de saberes, a través de la cual convergen elementos matemáticos y de las ciencias naturales, que buscan a través de conocimientos teóricos y prácticos, el desarrollo de habilidades para el aprovechamiento económico de los recursos y fuerzas de la naturaleza, de manera que se generen toda una serie de materiales que fomenten el mejoramiento de la salud humana (Enderle y Bronzino, 2012; Truskey, 2017). De allí el impacto que ha presentado durante los últimos años, a raíz de los nuevos descubrimientos en el campo de la biología, lo cual incide directamente sobre los sistemas sanitarios -y por ende los servicios de salud- a los cuales sirve esta profesión (Paina y Peters, 2012). Sólo por mencionar algunos de los hechos en que más se ha evidenciado la influencia de la ingeniería biomédica pueden citarse la decodificación del mapa del genoma humano, lo cual posibilita detectar de manera temprana la presencia de genes anormales e incluso modificar la estructura del ADN - ácido desoxirribonucleico- mismo y que pueden alterar la condición saludable de un sujeto (Haraksingh y Snyder, 2013); así como los avances afines a la nanotecnología y tecnologías de la información y la comunicación que cada día son más prometedores en el gerenciamiento de los sistemas de sanidad (Aziz y Akbarzadeh, 2017).

En este sentido, debe resaltarse la conexidad existente entre los procesos asociados a la ingeniería biomédica y la tecnología, afirmación que cobra validez en la medida que las constantes innovaciones tecnológicas asociadas al sector salud son fruto de los diversos hallazgos biomédicos, los cuales responden a una demanda latente del mercado para garantizar tratamientos oportunos; lo cual a su vez asigna un compromiso ético por parte de los profesionales, puesto que es bien sabido el riesgo existente en la implementación de estas tecnologías, por lo que su uso deberá condicionarse bajo la premisa que los beneficios asociados al paciente sean mayores que los riesgos a los que éste pudiese estar expuesto (Toscas y Toscas, 2015; Páez-Avilés et al. 2015). Consecuentemente con lo hasta aquí planteado, se presenta otro elemento que es de suma importancia, y se relaciona con el rol que ejercen las universidades en el campo, no sólo por la formación del talento humano, sino también por la consolidación de los procesos investigativos y tecnológicos de las sociedades a las cuales sirven, donde emerge una preocupación asociada a los grandes esfuerzos que se llevan a cabo para este tipo de procesos y la baja circulación del conocimiento generado (Ansuattigui et al. 2013; Urrestarazu, 2014), es decir, la inapropiada gestión de transferencia tecnológica efectuada.

La transferencia tecnológica es un concepto que adquiere cada vez mayor relevancia, con más razón en el marco de una sociedad globalizada y donde la innovación se ha convertido en el eje referencial de la dinámica social. Esta puede entenderse como el proceso que permite la transferencia de conocimientos técnicos, económicos y comerciales, entre una organización y otra, así como los aprendizajes que estimulan la capitalización del conocimiento generado, es decir, la aplicabilidad del mismo para configurarlo en beneficios económicos (Vila et al, 2013; Bozeman et al., 2015). En la actualidad, la definición del concepto es diversa, y puede comprenderse a través de tres enfoques: uno asociado al modelo de apropiación, a través del cual una vez generada la idea, ésta es puesta a disposición de la comunidad mediante informes y demás estrategias de divulgación. Otro, se vincula al modelo de difusión, el cual parte de la premisa que las innovaciones deberán difundirse con los usuarios potenciales de las mismas, de manera que el proceso de transferencia se dará de una manera natural y apropiada (Dearing et al.,2013); y el tercer enfoque, se asocia al modelo de conocimiento útil, que sin lugar a dudas es el que más ha logrado posicionarse en los últimos años, donde el nuevo conocimiento adquiere una connotación de utilidad universal, por lo cual el reto consistirá en comunicarlo a través de los canales adecuados, y en caso de no ser adoptado, la inconsistencia radicará en que el destinatario final no lo entiende (Martínez y Medina, 2013; López et al., 2015).

Este proceso adquiere por tanto gran relevancia para el campo de las ciencias biomédicas, en función del impacto que generan sobre la calidad de vida de los seres humanos, particularmente con los constantes desarrollos aportados desde la ingeniería biomédica (LeDuc et al, 2014). Algunos autores registran experiencias de transferencia tecnológica en la región, como en el caso referido por Perone et al (2013) sobre el estímulo generado desde el Institut Pasteur de Montevideo, que propende por ofrecer espacios en laboratorios de innovación para organizaciones públicas y privadas del MERCOSUR - Mercado Común del Sur-; así como el panorama de Brasil con el uso de estrategias biotecnológicas que le han permitido no sólo grandes desarrollos a nivel social y económico, sino que también han puesto en manifiesto el aprovechamiento de los conocimientos generados desde la academia (Caffé de Oliveira y Biondi, 2013). Para el caso colombiano, los estudios asociados a transferencia tecnológica son aún limitados, tal como lo demuestran Becerra et al., (2013) en su estudio, quienes ponen en evidencia la relevancia que tiene la promoción de políticas públicas que permitan la consolidación de los procesos de transferencia en el país, para garantizar la competitividad del mismo, lo cual sólo puede lograrse a través del fortalecimiento del capital relacional de los diversos agentes del sistema de ciencia, tecnología e innovación, donde la articulación entre las empresas e Instituciones de Educación Superior (IES) es vital. 
En este mismo sentido, en Colombia los procesos de transferencia tecnológica aplicada al sector salud son incipientes, y la evidencia de ello está en la escasa información existente al respecto. Por ello se propuso una investigación con la intencionalidad de adaptar un modelo de transferencia tecnológica existente en el medio a los sistemas de rehabilitación en ingeniería biomédica en la ciudad de Medellín para el año 2016.

\section{METODOLOGÍA}

Se propone una investigación con el objetivo de adaptar un modelo de transferencia tecnológica asociado a los sistemas de rehabilitación en ingeniería biomédica en la ciudad de Medellín (Colombia) para el año 2016, a través de un análisis de caso. Para ello se incluyeron 10 fundaciones con presencia en la ciudad de Medellín, para las cuales el criterio de selección radicó en el impacto generado a nivel social y; así mismo, se tomaron como participantes dos organizaciones especializadas en la elaboración de ayudas técnicas para los procesos de rehabilitación y una Institución de Educación Superior -IES- que trabajara en el campo disciplinar de la ingeniería biomédica a favor de la población en situación de discapacidad. Las fundaciones que cumplieron con los criterios de inclusión delimitados por el estudio fueron: Colombia Accesible, Corporación "Un ser feliz", el Comité de Rehabilitación, la Fundación ADA -apoyo, deportes, amigos-, Héroe Camina, HANDICAP International Colombia, Fundación CIREC, Laboratorios Gillette, el Instituto de Ortopedia Infantil Roosevelt y la Teletón.

Respecto a las organizaciones especializadas en la elaboración de técnicas para los procesos de rehabilitación, se contó con la participación de Accesibilidad Digital y Mahavir Kmina. En cuanto a la institución de educación superior seleccionada, se tomó al Instituto Tecnológico Metropolitano -ITM-, dado su trabajo articulado entre el campo disciplinar de la bioingeniería y las personas en situación de discapacidad. Una vez definidos los participantes del estudio de caso, el equipo investigador procedió con la definición de las técnicas e instrumentos para la recolección de la información, por lo que se diseñó una entrevista que permitiera obtener los datos necesarios para la construcción del modelo propuesto, con base en los diálogos sostenidos con los diferentes funcionarios de transferencia tecnológica en las instituciones participantes.

Cabe aclarar que para el contacto con las instituciones se tomaron en cuenta todas las consideraciones éticas garantes de la seguridad de los participantes, así como la confidencialidad respecto al uso de la información recolectada, catalogándose esta investigación como de riesgo mínimo a la luz de la Resolución 8430 de 1993, del ministerio de Salud, dado que no se modificarían estructuras físicas, psicológicas o sociales de los informantes de manera intencionada (Monterrosa, Márquez y Arteta, 2014), para ello al inicio de cada una de las entrevistas se entregó y se diligenció un consentimiento informado donde se manifiesta la intencionalidad del trabajo y las condiciones para la participación, así como el manejo que se daría a la información.

Bajo este panorama, para la construcción del modelo se partió del diagnóstico llevado a cabo en las instituciones participantes, donde el punto de partida del mismo se basa en las necesidades manifiestas por los pacientes y sus familiares desde el momento en que buscan medios para el mejoramiento de sus condiciones de vida a raíz de la discapacidad presentada; para ello las ideas que generen las soluciones adecuadas pueden provenir del ingenio institucional o de la búsqueda rigurosa de alguna tecnología en el campo que permita dar solución a la demanda implícita. Sin embargo, se identifica que existe una brecha entre la expresión de la necesidad y la solución del requerimiento, en la medida que las organizaciones desconocen el rol que puede desempeñar un ingeniero biomédico para el desarrollo de éstas. Adicionalmente cabe destacar que, del diagnóstico elaborado, se encontró que en ninguna de las instituciones participantes poseían oficinas encargadas de la transferencia tecnológica y que en éstas se identifica como barrera para dicha transferencia los constantes trámites burocráticos existentes al interior de las organizaciones, lo cual ralentiza los procesos.

\section{PROPUESTA DE MODELO}

Con base en el diagnóstico preliminar que se llevó a cabo con las instituciones participantes, y teniendo en cuenta las falencias existentes en el sector de rehabilitación, se propuso un modelo de transferencia tecnológica con aplicabilidad a dicho sector, en el cual se pretende mejorar el proceso, enmarcándolo dentro de las políticas de las instituciones y mediante la adecuación del modelo dinámico de transferencia tecnológica que se explica a continuación.

El modelo dinámico, se consolida como una versión mejorada del lineal, y tiene por objetivo la transferencia de conocimientos a través de su difusión, identificando una serie de aspectos que suelen pasar inadvertidos a la hora de transferir nuevos saberes, entre ellos se destaca: la comprensión intercultural, las habilidades de negociación y los incentivos para la investigación. Igualmente, ha de mencionarse el origen que tiene 
este modelo con el concepto de relacionamiento entre las empresas, las universidades y el Estado, que adquiere cada vez una connotación más relevante para los procesos de gestión del conocimiento (Arias y Aristizábal, 2011; Pineda, 2013). La figura 1 presenta la estructura del modelo dinámico de transferencia del conocimiento.

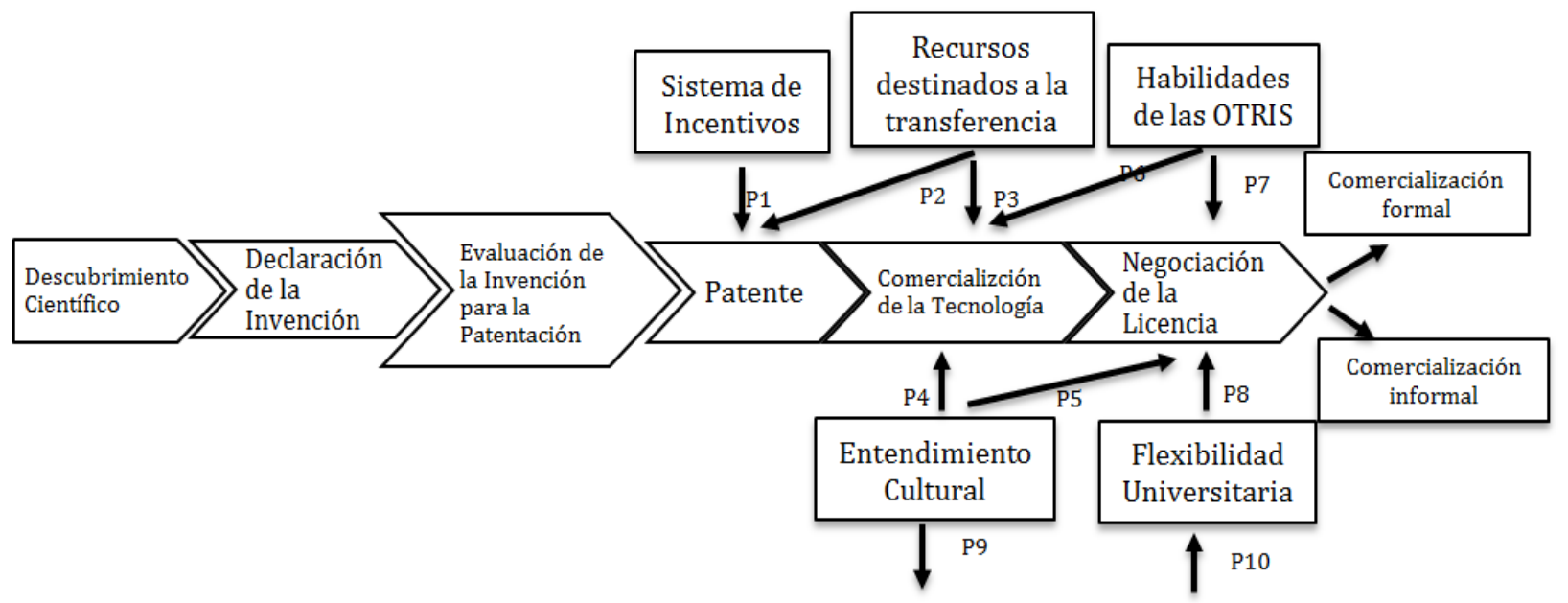

Fig.1: Modelo Dinámico de Transferencia (Tomado de Arias y Aristizabal, 2011).

Por tanto, se tomó el modelo dinámico de transferencia como referente para la adaptación efectuada por los investigadores, en la medida que se incluyen una serie de facilitadores que permiten que el conocimiento generado en la academia, pueda realmente fluir hacia las instituciones o clientes potenciales, en este caso a las fundaciones cuyas acciones se dirigen a las personas en situación de discapacidad y el mejoramiento de su calidad de vida. En la figura 2 se muestra un modelo de transferencia tecnológica para sistemas de rehabilitación en ingeniería biomédica.

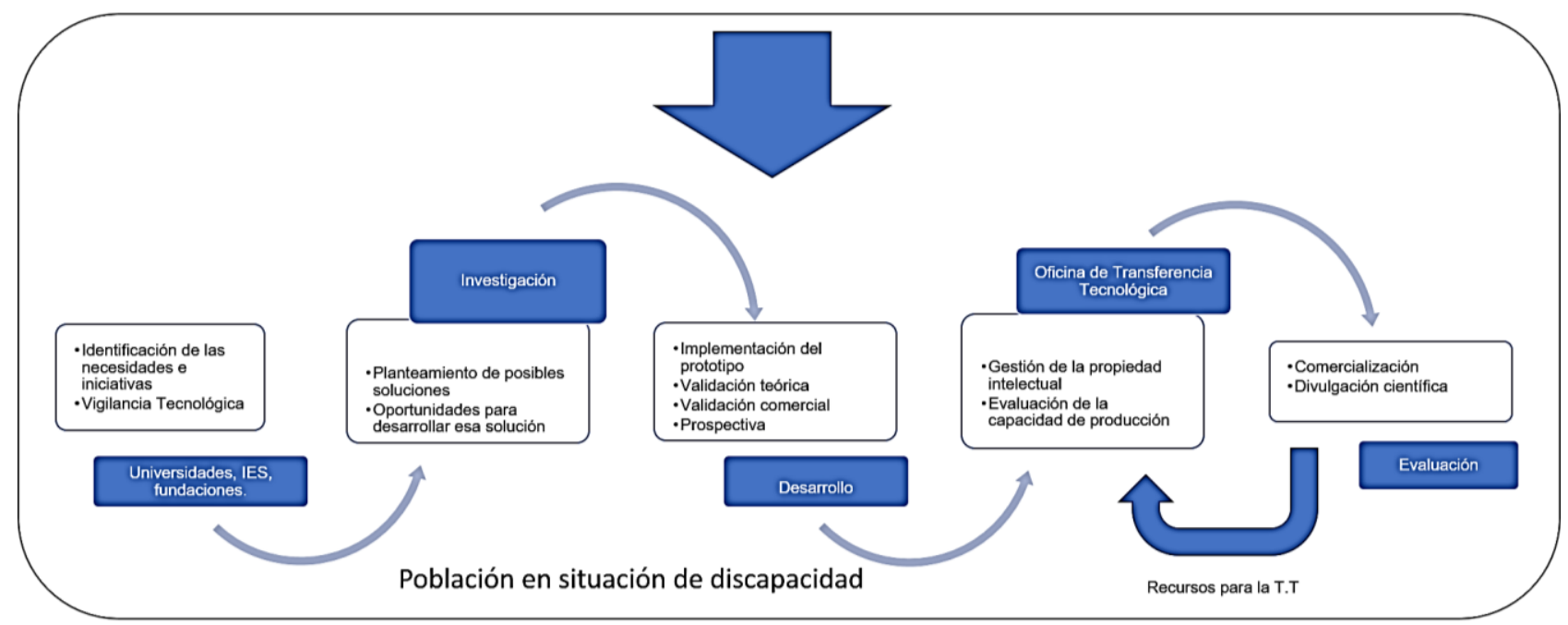

Fig. 2: Adaptación de un modelo de transferencia tecnológica para sistemas de rehabilitación en ingeniería biomédica.

El modelo propuesto tiene como objetivo la transferencia de tecnología -T. T- mediante la comercialización y divulgación científica, para impactar de manera positiva la población con discapacidad. Para que se lleve a cabo esta transferencia, el modelo debe estar respaldado en todas sus divisiones, con recursos tecnológicos y de personal. Los recursos tecnológicos pueden estar contemplados como software para la implementación de la vigilancia tecnológica, herramientas tecnológicas y equipos para el desarrollo de la investigación e implementación de prototipos.

En la parte del desarrollo de esa transferencia y después de tener el prototipo real de la solución que se vaya a brindar, se propone realizar un ejercicio de prospectiva. Tener el elemento o la solución real, puede generar una mejor visión a futuro de lo que podrá suceder con el prototipo y las mejoras que se podrían realizar para que tenga un mayor impacto en el mercado. Después del ejercicio de prospectiva y de la retroalimentación con las mejoras, se debe realizar una validación técnica que contempla la validación del prototipo (esta es la parte más costosa en tiempo, materiales y en personal) y luego una comercial para determinar el éxito de la solución propuesta en el mercado. Una vez desarrollado este paso dentro del modelo, pero sin pasar al siguiente, se proponen unos incentivos al equipo de investigación y desarrollo. 
Se propone en este punto del modelo porque aquí se termina el proceso con el equipo, ya que, luego de este paso, el prototipo es estregado a la Oficina de Transferencia de Tecnología -OTT-, quienes se encargarán de la realización y comercialización de las licencias de patentes y los permisos de comercialización. Los recursos para los miembros de la OTT deben estar enmarcados en los utilizados para la T.T dentro de la institución. Dependiendo del número de licencias que la oficina de T.T logre concretar se destinarán esos mismos fondos para sacar adelante otros procesos de transferencia de tecnología, es decir, la institución solo tendría que participar de manera inicial con recursos, una vez se logre comercializar algún producto, parte de las ganancias deben de ir destinadas a los demás procesos de T.T haciendo el modelo auto sostenible.

Para rehabilitar pacientes con discapacidad es importante que las soluciones propuestas cuenten con una optimización en tiempo del flujo del proceso. Es por esto que los ingenieros biomédicos están contemplados desde la fase inicial, en la detección de falencia y en la vigilancia tecnológica. En este modelo también se eliminan las barreras de la burocracia, esperando que los tiempos en los procesos no debiliten la transferencia de la ayuda técnica desde el momento de su creación hasta el impacto con el paciente. Se incluye la OTT para que ésta sea la encargada de mediar entre la universidad y la empresa, permitiendo así que los proyectos desarrollados en las universidades que imparten ingeniería biomédica lleguen a las organizaciones.

En lo que atañe a la comercialización y divulgación científica, ésta se encuentra enmarcada dentro de un proceso de evaluación. Es decir, se propone con el modelo una retroalimentación de parte de los clientes sobre el producto entregado, ya que se considera que el acompañamiento post-entrega de parte de la academia o de la empresa que desarrolla equipos para rehabilitación física, permitirá mejorar sus diseños de acuerdo al contexto en el que el paciente o el usuario se desarrollen.

\section{VALIDACIÓN DEL MODELO PROPUESTO}

La validación del modelo propuesto se realiza en tres etapas: una etapa teórica, un caso de estudio y dos casos de valoración por parte de expertos aplicados a la fundación El Comité de Rehabilitación y a la Oficina de Transferencia Tecnológica del Instituto Tecnológico Metropolitano. La validación teórica explica por qué se adecúa el modelo dinámico para este caso en específico, los dos casos de estudio podrían mostrar los impactos esperados al aplicar el modelo dentro de las instituciones correspondientes y se mide la percepción de la utilidad y viabilidad del modelo. Cabe resaltar que, dentro de la validación de este modelo, este no tuvo una aplicación donde se pudiera implementar en pacientes, debido a que ninguna de las instituciones encuestada tiene contratado ningún ingeniero biomédico para este fin, tampoco tienen departamento de investigación, ni convenios con las instituciones educativas para la utilización de la OTT de estas instituciones.

\section{Validación Teórica}

Las modificaciones realizadas al modelo lineal que realizan algunos autores para dar origen al modelo dinámico, se basan en los incentivos que deben aplicarse a los investigadores y desarrolladores del producto y a las Oficinas de Transferencia Tecnológica para que se generen más patentes y licencias, además de agilizar su comercialización en la industria. Esta parte del modelo dinámico se toma y se aplica en el modelo propuesto como incentivos en la etapa después del desarrollo y para las oficinas de transferencia tecnológica, debido a la situación económica de las instituciones públicas como el ITM, se establece que los recursos generados de la comercialización retroalimente los procesos que se realicen de manera consecutiva generando un bucle auto sostenible. Adicionalmente, debe acotarse que un modelo de transferencia tecnológica será exitoso siempre y cuando esté enmarcado dentro de las políticas de las instituciones en las cuales se va a aplicar y se tenga acceso por parte de las personas interesadas a la información y estructura que los soporta. La implementación y el posible éxito de este modelo dentro del ITM tiene condiciones a favor, como son el tener el pregrado en Ingeniería Biomédica, cuenta con oficina de discapacidad e inclusión social, una oficina de transferencia de tecnología y es una institución adscrita al municipio de Medellín, de donde provienen gran parte de los recursos para su sostenimiento.

\section{Validación Práctica: Silla de ruedas CATA}

Se realiza a través de la silla de ruedas CATA, como producto de la institución. Ésta nace de la necesidad de la población usuaria de sillas de ruedas que estudia en el ITM y que tenía necesidad de desplazarse dentro de la institución a la biblioteca. Debido a la topografía del lugar esta silla debió ser diseñada con ciertas especificaciones. El acercamiento para este caso se realizó desde el pregrado de Ingeniería de Diseño Industrial y un grupo de profundización adscrito a este programa que se llama Diseño y Salud. Éstos diseñaron una silla con un motor y una batería potente para enfrentarse a las condiciones del terreno e 
inicialmente el diseño solo fue pensado para las condiciones de la institución, es decir, solo podía ser utilizada dentro de la universidad. No se tiene conocimiento si se ha realizado un proceso de vigilancia tecnológica tal como lo propone el modelo, antes de implementar esta solución.

En la etapa de investigación se realiza un planteamiento de las posibles soluciones al problema enfrentado y se buscan oportunidades de desarrollar esta solución. En el caso de CATA se realizó mediante un proyecto presentado a una convocatoria interna. Luego se implementa el prototipo y se hace una validación técnica dentro de la institución, se realizan pruebas en las rampas con peralte habilitadas para usuarios de sillas de ruedas. No se tiene conocimiento de la realización de la validación comercial, ya que el prototipo solo estaba pensado para una población específica y tampoco se tiene conocimiento de haberse desarrollado un ejercicio de prospectiva donde se evaluarán las oportunidades de la herramienta tecnológica en un futuro. Tampoco se conoce si se realizó algún reconocimiento económico a manera de incentivos a los investigadores y desarrolladores. Posteriormente, el caso CATA es tomado por la Oficina de Transferencia Tecnológica de la institución.

Según el personal de la Oficina de Transferencia Tecnológica, CATA no tuvo una adecuada validación comercial ya que su comercialización está sesgada solamente a un contexto y a una necesidad, debido a esto la comercialización puede estar ligada a una licencia o a una Spin Off pero no a la realización de varios prototipos iguales para la venta directa. El conocimiento aplicado a este prototipo fue divulgado en los periódicos y en programas de televisión de la ciudad de Medellín. Aunque no se tiene conocimiento del seguimiento de todos los pasos enmarcados en el modelo propuesto, se identificaron falencias en algunos ítems como la validación comercial del prototipo, dando como resultado una incipiente comercialización del prototipo, afectando tanto a los usuarios de sillas de ruedas del instituto tecnológico metropolitano (ITM) como a potenciales clientes futuros fuera de la institución.

\section{Validación por experto: El Comité de Rehabilitación de Antioquia}

El modelo propuesto fue presentado ante el subdirector médico de "el Comité", durante la entrevista acerca de la percepción del modelo se tocan temas como el poco conocimiento a nivel general y el papel que un ingeniero biomédico puede realizar en una fundación donde se utilizan herramientas tecnológicas para la rehabilitación de pacientes. Respecto a la identificación de las necesidades se sugiere añadir el contexto del paciente a la hora de adquirir o de formular la posible solución para su discapacidad. Lo anterior teniendo en cuenta que el contexto de vida del paciente define el tipo de ayuda que requiere para su inclusión laboral o las modificaciones que habría que realizar sobre una herramienta tecnológica base como lo es por ejemplo una silla de ruedas. Por consiguiente, se debe identificar el requerimiento del usuario ligado a su contexto. En cuanto a la parte investigativa y al planteamiento de posibles soluciones, se dice que Medellín no cuenta con una empresa que se dedique exclusivamente a adaptar las ayudas técnicas y tecnológicas al contexto de cada paciente, estas modificaciones se realizan de manera empírica por personal de alguna empresa que desarrolla este tipo de tecnologías.

Cuando se evalúa el recuadro de desarrollo se presenta una discusión acerca de la validación comercial, ya que cada discapacidad es personal y única debido al contexto, ¿cuántas personas pueden comprar un prototipo desarrollado de forma personalizada? Como respuesta a la pregunta anterior se comenta que se debería implementar una herramienta tecnológica base que permita realizar modificaciones de acuerdo a cada persona con discapacidad y su contexto. Se presenta una opinión favorable acerca de lo incentivos en la parte de desarrollo del prototipo y se sugiere que éstos estén estipulados dentro del presupuesto de los proyectos desarrollados dentro de la fundación. Además, se sugiere adicionarle al modelo una flecha que simbolice la retroalimentación desde la parte de evaluación a la primera parte del acercamiento del Ingeniero Biomédico a la necesidad.

En cuanto a la aplicación del modelo de transferencia propuesto, se destacan las alianzas con las universidades que imparten la Ingeniería Biomédica, el acercamiento del ingeniero biomédico a esta problemática social para abrir campos de acción poco conocidos en el medio, la implementación del prototipo antes de la validación mecánica y comercial, paso que también resulta muy útil para realizar el trabajo de visión a futuro.

\section{DISCUSION FINAL}

La transferencia tecnológica se constituye sin lugar a dudas en un aspecto que va cobrando cada vez más peso en las denominadas sociedades del conocimiento, en la medida que se espera que realmente las diferentes innovaciones y resultados de investigaciones puedan circular en los colectivos donde son generadas, garantizando no sólo su socialización, sino también su aplicabilidad. 
Durante la realización de este estudio queda en evidencia la evolución del concepto y los diversos modelos existentes para llegar a su comprensión, donde el dinámico es el que más fuerza ha cobrado, dada la articulación de las universidades -generadoras de conocimiento-, el Estado -como ente rector de las naciones- y las empresas -como unidades productivas, generadoras de empleo y capital-, las cuales configuradas dan lugar a una perspectiva de desarrollo que trasciende la visión economicista, involucrando también elementos sociales, políticos y culturales.

Así mismo, queda manifiesta la incipiente evolución que ha tenido esta temática en el contexto colombiano, más aún cuando se refiere a los procesos de rehabilitación en el campo de la ingeniería biomédica, lo cual pone al país en una situación de desventaja en comparación con otros contextos y experiencias exitosas de la región. Por lo tanto, esta investigación permite validar el rol fundamental que juegan las universidades en el marco de una nación cada día más globalizada, al proveer nuevos desarrollos tecnológicos que impacten a aquellas poblaciones que, por sus condiciones físicas, psicológicas y sociales, aunadas a una serie de imaginarios de los colectivos, les han llevado al ostracismo y a tener una calidad de vida que va en contravía de las políticas estatales de inclusión.

Adicionalmente, se plantean retos también para la academia en el momento de crear modelos en el campo ingenieril, donde estos constructos precian contar con la participación de agentes externos como las empresas y el Estado, pues permiten que la construcción de los mismos y su posterior implementación a través de prototipos sea exitoso y acorde a las necesidades de las poblaciones a quienes vayan dirigidas, particularmente aquellas innovaciones asociadas a la rehabilitación de personas en situación de discapacidad, que requieren no sólo dar solución a la discapacidad manifiesta, sino también a una serie de condiciones endógenas y exógenas del individuo que garantizarán el mejoramiento de su calidad de vida, y por ende un adecuado proceso de transferencia tecnológica, al lograr que los conocimientos generados sean efectivamente comunicados y recibidos.

\section{CONCLUSIONES}

De acuerdo al análisis de la literatura, la propuesta del modelo y los resultados de este trabajo, se pueden obtener las siguientes conclusiones principales. Primera: Los modelos de T.T existentes son adaptables a cualquier ámbito tecnológico como se demostró en este caso, sin embargo, lo realmente importante es que dicho modelo este enmarcado dentro de las políticas de las instituciones que lo vayan a implementar. Segunda: Los cambios hechos al modelo, de acuerdo a lo percibido por el experto, generaran un impacto más positivo para los pacientes con discapacidad comparado con el cómo se realiza la T.T hoy. Tercera: Los resultados de las tres validaciones del modelo presentado concuerdan en la importancia y utilidad de la propuesta en cuanto a los incentivos a los investigadores y a la auto sostenibilidad de la OTT, al igual que en el valor de tener un prototipo físico del desarrollo tecnológico que se esté implementando. Cuarta: La metodología empleada para la recolección y el análisis de la información puede ser modificada aumentando la población muestra para lograr un diagnóstico más globalizado. Quinta: La implementación del modelo propuesto por parte de algunas fundaciones puede generar claridad acerca del tipo de tecnología que se está desarrollando y comprando para personas con discapacidad. Pero su implementación dentro de las instituciones requiere de un compromiso por parte de los directivos en cuanto a contratación del personal idóneo y los incentivos a investigación.

\section{REFERENCIAS}

Ansuattigui, R.V., Caulliraux-Pithon, A. J., y Fernandes, J.L., Prácticas de Gestión del Conocimiento en una Institución Pública de Investigación: El Caso del Centro Tecnológico del Ejército en Brasil (CTEx). Información Tecnológica, https://dx.doi.org/10.4067/S0718-07642013000500007, 24(5), 51-60 (2013)

Arias, J.E. y Aristizábal, C.A., Transferencia de conocimiento orientada a la innovación social en la relación ciencia-tecnología y sociedad, Pensamiento \& Gestión, 31, 137 - 166 (2011)

Aziz, S.G., Aziz, S.G. y Akbarzadeh, A., Advances in Silver Nanotechnology: An Update on Biomedical Applications and Future Perspectives. Drug Research. 2017 Apr., 67(4), 198-203. doi: http://dx.doi.org 10.1055/s-0042-112810. Epub 2017 Feb 16 (2017)

Becerra, F.; Serna, H.M. y Naranjo, J.C., Redes empresariales locales, investigación y desarrollo e innovación en la empresa. Cluster de herramientas de Caldas, Colombia, doi: http://dx.doi.org/10.1016/j.estger.2013.05.013, Estudios Gerenciales, 29 (127), 247 - 257 (2013)

Bozeman, B., Rimes, H., y Youtie, J., The evolving state-of-the-art in technology transfer research: Revisiting the contingent effectiveness model, Research Policy, 44(1), 34-49 (2015) 
Caffé de Oliveira, D. y Biondi, I., Análisis de nivel subnacional de Política de Desarrollo de Biotecnología en Brasil: Innovación tecnológica y la salud humana en el Estado de Bahía, Journal of Technology Management \& Innovation, http://dx.doi.org/10.4067/S0718-27242013000300032, 8 (1), 26 - 38 (2013)

Dearing, J., Smith, D., Larson, R., y Estabrooks, C., Designing for Diffusion of a Biomedical Intervention. American Journal of Preventive Medicine, http://dx.doi.org/10.1016/j.amepre.2012.09.038, 44(1), 70-76 (2013)

Enderle, J.D. y Bronzino, J.D., Introduction to biomedical engineering, Third Edition, 16 - 17, Academic Press, Burlington MA, United States of America (2012)

Haraksingh, R. R., y Snyder, M. P., Impacts of variation in the human genome on gene regulation. Journal of molecular biology, 425(21), 3970-3977 (2013)

LeDuc, P., Agaba, M., Cheng, C., Gracio, J., Guzman, A., y Middelberg, A., Beyond Disease, How Biomedical Engineering Can Improve Global Health. Science Translational Medicine, http://dx.doi.org/10.1126/scitranslmed.3009067, 6(266), 1-3 (2014)

López, D., Marulanda, C. E. y López, M., Métricas de Valoración de la Gestión del Conocimiento para las Pequeñas y Medianas Empresas del Sector Tecnologías de Información en el Triángulo del Café en Colombia. Inf. Tecnológica, https://dx.doi.org/10.4067/S0718-07642015000300020, 26(3), 173-183. (2015)

Martínez, K.I y Medina, M.E., Transferencia tecnológica en el área de adicciones: El programa de intervención breve para adolescentes, retos y perspectivas. Salud Mental, 36 (6), $505-512$ (2013)

Monterrosa, A.; Márquez, J. y Arteta, C., Disfunsión sexual en mujeres climatéricas afrodescendientes del Caribe Colombiano. latreia, 27 (1), $31-41$ (2014)

Páez-Avilés, C., Juanola-Feliu, E., Bogachan-Tahirbegi, I., Mir, M., González-Piñero, M., y Samitier, J. Innovation and technology transfer of medical devices fostered by cross-disciplinary communities of practitioners. International Journal of Innovation Management, http://dx.doi.org/10.1142/s1363919615400125, 19(06), 1-27 (2015)

Paina, L., y Peters, D.H., Understanding pathways for scaling up health services through the lens of complex adaptive systems. Health policy and planning, 27(5), 365-373 (2012)

Perone, M., G. Velásquez y otros siete autores., Primera Red Sudamericana de Biomedicina: Investigación, educación y biotecnología aplicadas a la salud, (Artículo Ocasional), ISSN: 0025-7680, Medicina, Buenos Aires, 73 (1), $75-77$ (2013)

Pineda, L., Prospectiva estratégica en la gestión del conocimiento: una propuesta para los grupos de investigación colombianos. Investigación y Desarrollo, 21 (1), 237 - 259 (2013)

Toscas, F.S. y Toscas, F., El sobrediagnóstico y sus implicaciones en la Ingeniería Clínica. Revista Bioética, doi: http://dx.doi.org/10.1590/1983-80422015233090, 23 (3), 535 - 541 (2015)

Truskey, G.A., The Future of Biomedical Engineering, Current Opinion in Biomedical Engineering, doi: http://dx.doi.org 10.1016/j.cobme.2017.04.004. (2017)

Urrestarazu, M., La importancia de la investigación y la transferencia tecnológica local. Idesia, doi: http://dx.doi.org/10.4067/S0718-34292014000200001, 32 (2), 3 - 4 (2014)

Vila, M.F.; Guagliano, L.M.; Galante, O y Arciniega, A.A, Transferencia de tecnologías a una cooperativa en Argentina: Un estudio de casos, Journal of Technology Management \& Innovation: doi: http://dx.doi.org/10.4067/S0718-27242013000300018, 8 (1), 197 - 207 (2013) 